\title{
Protocolo de manejo del intento suicida en el Hospital del Salvador en Santiago de Chile Suicide attempt management protocol in Hospital del Salvador in Santiago de Chile
}

\author{
Juan Pablo Cárdenas B. ${ }^{1}$, Diego Santelices H. ${ }^{1}$, Arturo Fredes B. ${ }^{1}$ y Ramón Florenzano U. ${ }^{1,2}$
}

\begin{abstract}
Suicide is a global public health and social problem. It has important emotional consequences for the relatives of the deceased, as well as economic and emotional consequences for the rest of the community. Herein lies the importance of the early detection of potential cases and of utilizing adequate interventions. The present protocol was organized as a way of standardizing and giving the appropriate hierarchy to all interventions practiced in patients with suicidal risk within Hospital del Salvador.
\end{abstract}

Key words: Suicide; suicidal ideation; suicide, attempted. Rev Chil Neuro-Psiquiat 2012; 50 (4): 249-254

\section{Introducción}

$\mathrm{E}^{\mathrm{l}}$ suicidio es un problema que ha ido creciendo en importancia en las últimas décadas. El año 2000, aproximadamente un millón de personas fallecieron por suicidio, lo que corresponde a una tasa global de 16 por 100.000 habitantes ${ }^{1}$, teniendo el sexo masculino una probabilidad 5 veces mayor de consumarlo ${ }^{2}$. Está entre las 3 principales causas de muerte en adolescentes en el mundo, al menos 90.000 (hasta los 19 años de edad) fallecen por suicidio cada año ${ }^{3}$, y en Chile la tasa aumentó de 6,2 x 100.000 hab. el año 1997 a 10,8 el año $2004^{4}$. A partir de estas cifras quisimos elaborar un protocolo para el manejo de estos pacientes.

Es importante distinguir entre factores de riesgo y predicción de riesgo, ya que generalmente los primeros se evalúan en la población como factores aislados. La predicción en cambio, requiere de una atención especial a la manera en que los factores de riesgo interactúan entre sí en un determinado paciente, resultando esto en que iguales factores tienen valores subjetivos diferentes según quien consulta. Por lo anterior, la predicción no puede ser llevada a cabo meramente adicionando los distintos factores de riesgo. Hay diversos factores que predisponen a las personas al suicidio ${ }^{5}$, siendo de los más importantes las tentativas previas. El fallecimiento o suicidio de seres queridos, así como otras experiencias de gran adversidad también cobran importancia, y el aislamiento por sí solo puede ser considerado como motivo de hospitalización, dependiendo del caso. La comorbilidad psiquiátrica imprime a estos pacientes características particulares ${ }^{6}$. En un estudio llevado a cabo en nuestro Servicio se concluyó que los pacientes bipolares tienden a ser de menor edad, tienen trabajos menos estables y son de mayor riesgo suicida ${ }^{7}$.

Recibido: 22/08/2012

Aprobado: 12/12/2012

\footnotetext{
Servicio de Psiquiatría, Hospital del Salvador.

2 Facultad de Psicología, Universidad del Desarrollo.
} 
Debe destacarse que la mayoría de los pacientes psiquiátricos no intentan o consuman el suicidio. La dualidad con abuso de sustancias, al igual que la comorbilidad, influye enormemente: de las personas que cometen suicidio, del $25 \%$ a $50 \%$ han consumido alcohol antes de quitarse la vida ${ }^{8}$.

Entre los factores protectores, el adecuado establecimiento de una alianza terapéutica es central, ya que facilita la entrega de información y da al paciente una sensación de esperanza y conexión. En la medida que el tratamiento avanza el lazo se va fortaleciendo y profundizando. La cantidad y veracidad de la información entregada, está en gran medida relacionada con la confianza que la persona tiene en su tratante 9 . En adolescentes se ha visto que la aceptación de los padres puede ser un factor importante $\mathrm{i}^{10,11}$.

Respecto de la religiosidad, en nuestros estudios las pacientes depresivas con riesgo suicida son menos religiosas que la población general chilena ${ }^{12,13}$.

\section{Metodología}

Se realizó una búsqueda en Medline y en la Database Cochrane utilizando los términos "suicide", "suicidal ideation" y "suicide, attempted". Se seleccionaron los artículos más pertinentes, tanto originales como guías clínicas, preferenciando a aquellos en que se evaluaban distintas estrategias de intervención. Se buscaron especialmente publicaciones chilenas y de la información entregada, así como del consenso clínico entre los especialistas de los Servicios de Psiquiatría y de Urgencias del Hospital del Salvador, un hospital general en Santiago de Chile, se preparó un documento de trabajo que fue revisado por los encargados de preparación de protocolos clínicos en dicho Hospital. Este trabajo expone las principales recomendaciones del protocolo.

\section{Resultados}

\section{Evaluación en Servicio de Urgencia y derivación}

$\mathrm{Al}$ ingreso, se debe solicitar al entrevistado que entregue todos los objetos con que podría hacerse daño (armas, cinturones, cordones de zapatos, etc.) y los medicamentos que tiene a su disposición. A continuación, debe conducirse al paciente a una sala en que no haya materiales potencialmente dañinos, y donde sea posible observarlo. La evaluación debe proceder lo más ágilmente posible, y en caso que el paciente quiera retirarse antes de que ésta se complete, se debe considerar la pertinencia de medidas de contención física y/o farmacológica. Son importantes los instrumentos estructurados para no dejar fuera datos cruciales de la historia clínica cuando éstos no son utilizados ${ }^{14}$. Un examen físico completo es de regla en aquellas personas en las que se sospeche intoxicación, sobredosis o daño físico general. Cabe destacar que toda manifestación de intenciones suicidas debe ser tomada en serio y explorada a fondo por el equipo: quienes consuman el suicidio frecuentemente han manifestado ideación suicida $\mathrm{u}$ otros signos de alerta a familiares o profesionales de la salud ${ }^{15}$.

$\mathrm{Al}$ ser completada la evaluación, debe iniciarse el tratamiento médico indicado. El protocolo del Servicio de Urgencia del Hospital del Salvador (SUHDS) dispone de las siguientes alternativas:

- Pacientes sin daño físico, que no requieren de tratamiento médico, cuya evaluación para riesgo suicida es baja, y que tienen apoyo social adecuado, son enviados a su domicilio acompañados por un familiar responsable y con interconsulta para atención ambulatoria en el centro de la Red de Salud Mental del SSMO que corresponda según domicilio y nivel de complejidad.

- Pacientes psicóticos, con riesgo suicida elevado y/o red de apoyo social pobre pero sin necesidad de cuidados médicos, son hospitalizados en el Servicio de Psiquiatría. Los pacientes en riesgo vital o con necesidad de cuidados médicos, permanecen en observación y tratamiento en el SUHDS y pueden ser hospitalizados en otros servicios médicos o quirúrgicos según necesidad, siempre con interconsulta a la Unidad de Psiquiatría de Enlace.

- Ocasionalmente por solicitud del paciente o la familia se hacen derivaciones a centros privados, con contacto telefónico previo con el médico el tratante. 
La sedación debe evitarse en pacientes que hayan ingerido sobredosis de medicamentos psicótropos. Algunos casos requieren sedación, especialmente aquellos con elevados niveles de angustia o con franca agitación. Esta medida no constituye tratamiento para las condiciones psicológicas de base, se puede indicar de urgencia en forma ocasional

\section{Evaluación en el Servicio de Psiquiatría}

La literatura señala que la gran mayoría de los casos de intento suicidio presentan psicopatología. Por este motivo el manejo de la comorbilidad psiquiátrica o de la patología dual, con abuso de sustancias es fundamental.

La entrevista con familiares puede aportar datos útiles como el estado de ánimo habitual del paciente, si se siente o no esperanza de resolver los conflictos psicosociales, que son frecuentes como factor gatillante. La frecuencia de consumo de alcohol y/o drogas debe ser también indagada entre los familiares, ya que el paciente muchas veces la minimiza.

La clasificación en categorías de riesgo requiere de juicio clínico cuidadoso, pues el riesgo puede variar enormemente en plazos cortos y la asignación de personas a categorías estadísticas de riesgo no es predictiva de conducta suicida ${ }^{15}$. De hecho la mayor parte de los pacientes evaluados en servicios de salud mental (no necesariamente hospitalizados) y que posteriormente cometieron suicidio fueron catalogados como casos de riesgo bajo o nulo en el último contacto con el sistema de salud ${ }^{15}$.

Cada factor de riesgo específico es teñido por el contexto psicosocial. Es importante detectar los factores gatillantes de la conducta suicida. Son estos gatillantes, junto con el examen mental al momento de consultar, los componentes más relevantes ${ }^{16}$.

Al finalizar la estimación de riesgo debe elaborarse un plan de tratamiento, que debe incluir tres factores básicos:

- Asegurar la integridad física de la persona: el plan de tratamiento debe intentar evitar que la persona en crisis consume su intención. Esta intencionalidad fluctúa, por lo que debe ser monitorizada periódicamente.
- Establecer una relación terapéutica lo más sólida posible, del paciente con el entrevistador, o preferentemente con el sistema asistencial, ya que en muchos casos quien entrevista no será el tratante definitivo.

- Instalar tratamiento adecuado de los cuadros psiquiátricos de base y abordar cualquier estresor que haya precipitado el estado actual, de tal forma que cuando se de al paciente de alta hospitalaria, la situación del paciente haya mejorado. Si el paciente es dado de alta sin la correspondiente mitigación de los estresores que gatillaron el cuadro, el riesgo suicida debe ser observado cercanamente.

En el SPHDS, la decisión de hospitalizar se basa en aspectos tales como necesidad de manejo médico o psiquiátrico intensivo (p. ej. en psicosis agudas), planificación del intento suicida, historia de conducta impulsiva y red de apoyo social pobre. Las hospitalizaciones cortas no disminuyen el riesgo suicida, por lo que estos pacientes deben permanecer en el hospital hasta que la evaluación de riesgo lo indique $^{17}$. Los trabajos del SPHDS muestran que la hospitalización disminuye importantemente el riesgo suicida $^{18}$, no lo elimina en su totalidad, y se han descrito casos de suicidios consumados en unidades de hospitalizados, particularmente durante la primera semana de internación ${ }^{19}$. Posterior al alta, el primer control ambulatorio debiera realizarse durante la primera semana, considerando que es este el período de mayor riesgo finalizando la hospitalización ${ }^{15,19}$.

Respecto del tratamiento farmacológico, no existe evidencia suficiente que permita asociar de manera causal a los ISRS en general y a la fluoxetina en particular con un aumento del riesgo de suicidio ${ }^{20}$. Sin embargo, el riesgo podría aumentar paradójicamente luego del inicio del tratamiento antidepresivo, al mejorar la activación motora del paciente sin mejoría del ánimo. Las benzodiazepinas podrían estar asociadas a riesgo suicida al acentuar la sintomatología depresiva y/o desinhibir a los usuarios, haciéndolos por lo tanto, más propensos a actuar de manera impulsiva. Este riesgo es especialmente alto en casos con patología dual o historia de tendencias adictivas. 


\section{Conclusiones}

La evaluación del riesgo suicida es compleja, puesto que exige la integración de múltiples factores que oscilan de persona a persona. Su carácter fluctuante, así como las variaciones en el contexto psicosocial, puede inducir a errar. La investigación en este campo se encuentra limitada por tamaños muestrales pequeños y grupos diagnósticos heterogéneos, por lo que se requieren más estudios para elaborar estrategias más eficaces en la prevención, y así poder mejorar la calidad de cuidados requerida. En la situación actual, una de las más efectivas y accesibles herramientas de que dispone el tratante es el adecuado establecimiento de una alianza terapéutica, que facilita las estimaciones del riesgo, y mejora la adherencia a tratamiento. Esto, asociado a tratamientos enérgicos de los cuadros psiquiátricos de base y al abordaje oportuno de los principales estresores en la vida del paciente, en un marco que privilegie la integridad física se constituyen en el eje de un adecuado manejo del riesgo suicida.

En futuras comunicaciones esperamos adjuntar datos obtenidos a partir de la aplicación de este protocolo en nuestro centro para su análisis y discusión.

Anexo. Flujogramas

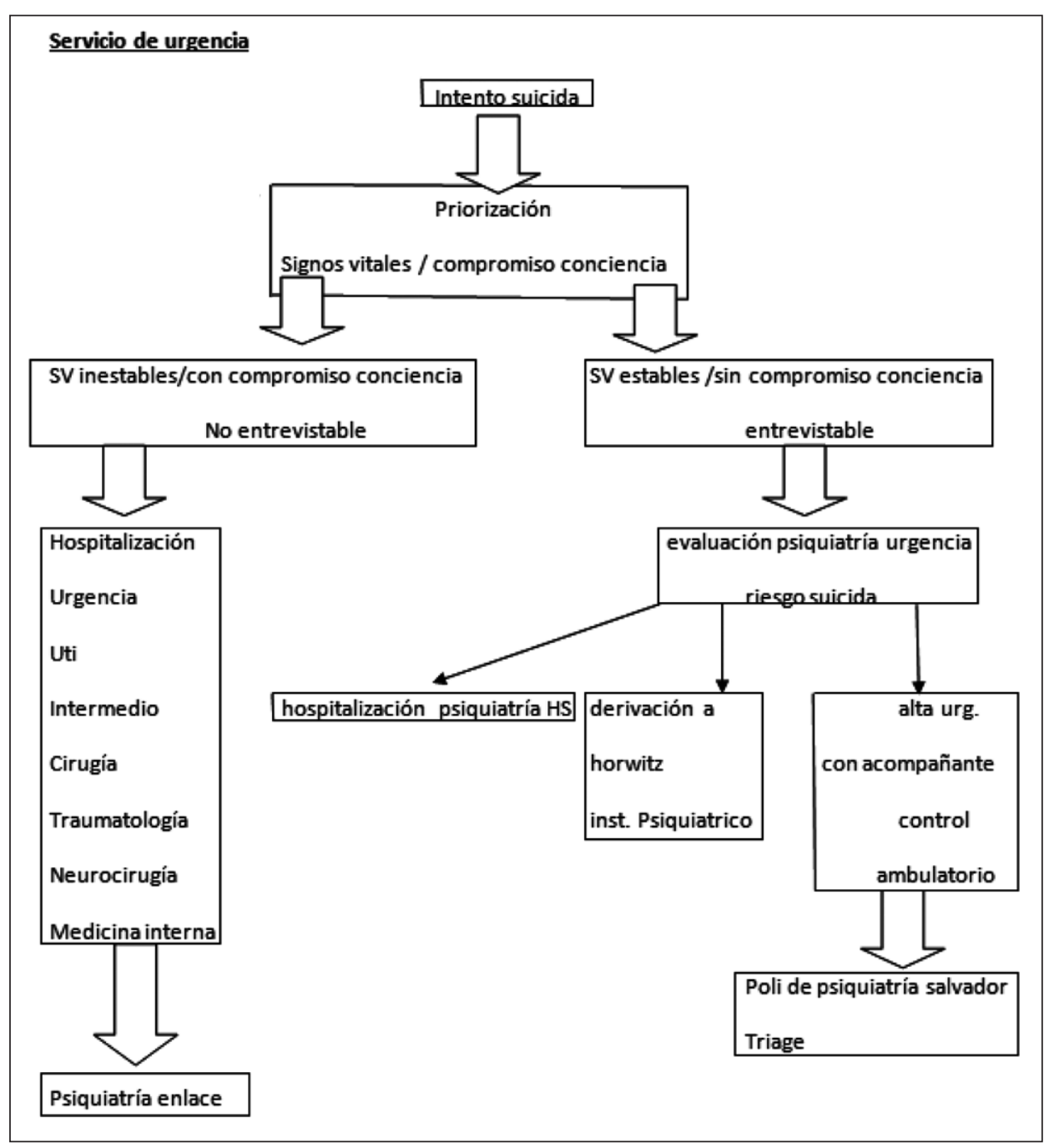




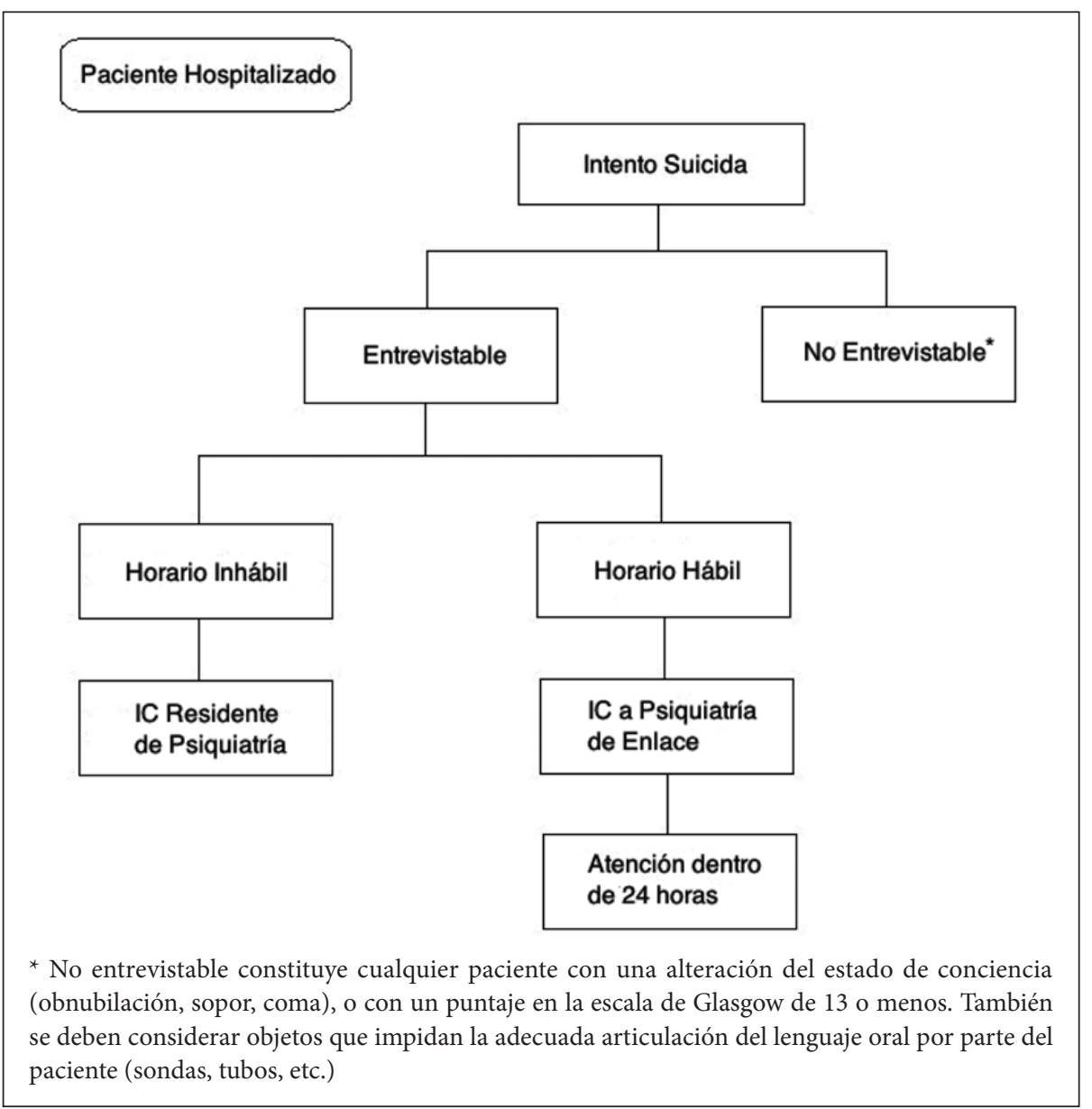

\section{Resumen}

El suicidio es un problema social y de salud pública en todo el mundo. Acarrea importantes consecuencias emocionales para las personas cercanas al fallecido y también consecuencias económicas y emocionales para el resto de la comunidad. De ahí la importancia de detectar tempranamente los potenciales casos y utilizar intervenciones adecuadas. El presente protocolo se estructuró como una manera de estandarizar y jerarquizar las intervenciones entregadas a pacientes con riesgo suicida en el Hospital del Salvador.

Palabras clave: Suicidio, ideación suicida, intento suicida.

\section{Referencias bibliográficas}

1. Organización Mundial de la Salud. Prevención del suicidio (SUPRE). Genova: OMS, 2009. Disponible en: http://www.who.int/mental_health/ prevention/suicide/suicideprevent/en/
2. World Health Organization. Global Burden of Disease: 2004 Update. Disponible en URL: http:// www.who.int/healthinfo/global_burden_disease/2004_report_update/en/index.html

3. Greydanus DE, Calles JJ. Suicide in children and adolescents. Prim Care 2007; 34: 259-73. 
4. Rojas I. Plan nacional de prevención del suicidio. Departamento de salud mental. MINSAL Chile 2008. Ponencia presentada en Seminario sobre Suicidio, Centro de extensión Universidad Católica, Santiago, Chile.

5. Florenzano R, Labra JF, Fasani R, San Juan K, Reynal J, Quevedo Y. Los Pacientes suicidas y para-suicidas pueden ser adecuadamente diagnosticados y tratados en una red pública de atención en salud mental. Revista Gaceta Psiquiatría Universitaria 2007; 3: 331-9.

6. Gómez, A. Trastornos depresivos y suicidalidad. Rev Chil Neuro-Psiquiat 1998; 36: 93-101.

7. Florenzano R, Leighton C, Retamal P, Osorio C, Gillibrand R, Tolosa P. Factores psicosociales asociados con suicidalidad, severidad y resultados del tratamiento en el trastorno afectivo bipolar. Trastornos del Ánimo 2008; 4: 88-94.

8. Brent DA, Perper JA, Moritz G, et al. Psychiatric risk factors for adolescent suicide: A case control study. J Am Acad Child Adolesc Psychiatry 1993; 3: $521-9$

9. Pinto A, Whisman MA, Conwell Y. Reasons for living in a clinical sample of adolescents. Journal Adolesc 1998; 21: 397-405.

10. Florenzano R, Cáceres C, Valdés M, Calderón S, Santander S, Casassus M. Conductas de Riesgo, síntomas depresivos, auto y heteroagresión en una muestra de adolescentes escolarizados en la Región Metropolitana de Santiago de Chile, 2007. Rev Chil Neuro-Psiquiat 2009; 47: 24-33

11. Florenzano R, Valdés M, Cáceres E, Casassus M, Sandoval A, Santander S, et al. Percepción de la Relación Parental entre Adolescentes Mayores y Menores de 15 años. Rev Chil Pediatr 2009; 80: 520-7.

12. Florenzano, R. Religious Beliefs and Clinical Severity among Suicidal Women Hospitalized in Chile. 42th Annual Meeting of the Society for Psychothe-

\section{Correspondencia:}

Dr. Diego Santelices H.

Servicio de Psiquiatría, Hospital del Salvador.

Fono: 02-5754083

E-mail: santelicesdiego@hotmail.com, rapy Research, Universidad de Berna, Suiza, 29 de Junio a 2 de Julio, 2011.

13. Florenzano R, Aspillaga C, Musalem, C. Alliende L, Cataldo E, Aratto C. Análisis retrospectivo de la evolución de mujeres depresivas con riesgo suicida tratadas en un servicio público de Psiquiatría en Santiago de Chile: El rol de la religiosidad. Actas del LXV Congreso Chileno de Neurología, Psiquiatría y Neurocirugía, Coquimbo, Chile 2010

14. Burn WK, Edwards JG, Machin D. Improving house physicians' assessments of self-poisoning. Br J Psychiatry 1990; 157: 95-100.

15. Appleby L, Shaw J, Amos T, McDonnel R, Harris C, McCann K, et al. Suicide within 12 months of contact with mental health services: National clinical survey. BMJ 1999; 318: 1235-9.

16. Beautrais AL, Joyce PR, Mulder RT. Precipitating factors and life events in serious suicide attempts among youths aged 13 through 24 years. J Am Acad Child Adolesc Psychiatry 1997; 36: 1543-51.

17. Van der Sande R, Van Rooijen L, Buskens E, Allart E, Hawton K, Van der Graaf Y, et al. Intensive in-patient and community intervention versus routine care after attempted suicide. A randomised controlled intervention study. Br J Psychiatry 1997; 170: 35-41.

18. Florenzano R, Gillibrand R, Oteíza C, Bruzzone C, Labra JF. Internación: Elementos pronósticos en pacientes con riesgo suicida hospitalizados en una unidad psiquiátrica de hospital general, 2004-2007. Actas del LXIII Congreso Chileno de Neurocirugía, Psiquiatría y Neurocirugía, 10-12 de Octubre de 2008, Viña del Mar.

19. Jacobson $\mathrm{G}$. The inpatient management of suicidality. In: Jacobs DG editor. The Harvard Medical School guide to suicide assessment and intervention. San Francisco: Josey-Bass; 1999. p. 383-405.

20. Jick SS, Dean AD, Jick H. Antidepressants and suicide. BMJ 1995; 310: 215- 8. 